\title{
The Quest For Highly Accurate Excitation Energies: A Computational Perspective
}

\author{
Pierre-François Loos, ${ }^{* \dagger}$ Anthony Scemama, ${ }^{\dagger}$ and Denis Jacquemin*, ${ }^{* \dagger}$ \\ $\dagger$ Laboratoire de Chimie et Physique Quantiques, Université de Toulouse, CNRS, UPS, France \\ $\ddagger$ Université de Nantes, CNRS, CEISAM UMR 6230, F-44000 Nantes, France \\ E-mail: loos@irsamc.ups-tlse.fr; Denis.Jacquemin@univ-nantes.fr
}

\begin{abstract}
We provide an overview of the successive steps that made possible to obtain increasingly accurate excitation energies with computational chemistry tools, eventually leading to chemically accurate vertical transition energies for small- and medium-size molecules. First, we describe the evolution of ab initio methods employed to define benchmark values, with originally Roos' CASPT2 method, then the CC3 method as in the renowned Thiel set, and more recently the resurgence of selected configuration interaction methods. The latter method has been able to deliver consistently, for both single and double excitations, highly accurate excitation energies for small molecules, as well as medium-size molecules with compact basis sets. Second, we describe how these high-level methods and the creation of representative benchmark sets of excitation energies have allowed to assess fairly and accurately the performance of computationally lighter methods. We conclude by discussing the future theoretical and technological developments in the field.
\end{abstract}

\section{Graphical TOC Entry}

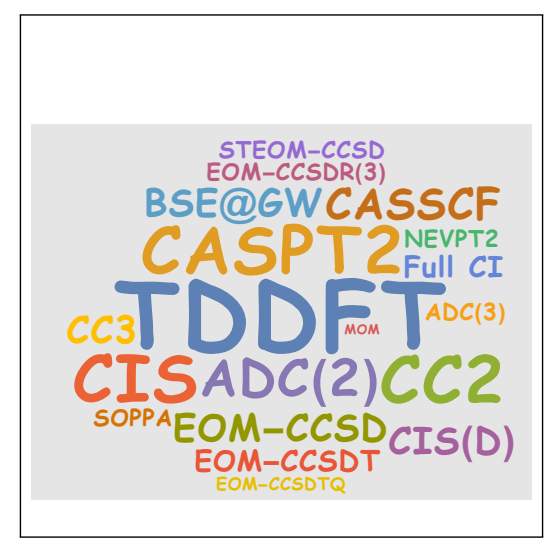

The accurate modeling of excited-state properties with $a b$ initio quantum chemistry methods is a clear ambition of the electronic structure theory community that will certainly keep us busy for (at the very least) the next few decades to come (see, for example, Refs. 1-3 and references therein). Of particular interest is the access to precise excitation energies, i.e., the energy difference between ground and excited electronic states, and their intimate link with photophysical and photochemical processes. The factors that makes this quest for high accuracy particularly delicate are very diverse.

First of all (and maybe surprisingly), it is, in most cases, tricky to obtain reliable and accurate experimental data that one can straightforwardly compare to theoretical values. In the case of vertical excitation energies, i.e., excitation energies at a fixed geometry, band maxima do not usually correspond to theoretical values as one needs to take into account both geometric relaxation and zero-point vibrational energy motion. Even more problematic, experimental spectra might not be available in gas phase, and, in the worst-case scenario, no clear assignment could be made. For a more faithful comparison between theory and experiment, although more computationally demanding, the so-called $0-0$ energies are 
definitely a safer playground. ${ }^{4-7}$

Second, developing theories suited for excited states is usually more complex and costly than their ground-state equivalent, as one might lack a proper variational principle for excited-state energies. As a consequence, for a given level of theory, excited-state methods are usually less accurate than their ground-state counterpart, potentially creating a groundstate bias that leads to inaccurate excitation energies.

Another feature that makes excited states particularly fascinating and challenging is that they can be both very close in energy from each other and have very different natures ( $\pi \rightarrow \pi^{*}, n \rightarrow \pi^{*}$, charge transfer, double excitation, valence, Rydberg, singlet, triplet, etc). Therefore, it would be highly desirable to possess a computational method (or protocol) that provides a balanced treatment of the entire "spectrum" of excited states. We think that, at this stage, none of the existing methods does provide such a feat at an affordable cost for chemically-meaningful compounds.

What are the requirement of the "perfect" theoretical model? As mentioned above, a balanced treatment of excited states with different character is highly desirable. Moreover, chemically accurate excitation energies (i.e., with error smaller than $1 \mathrm{kcal} / \mathrm{mol}$ or $0.043 \mathrm{eV}$ ) would be also beneficial in order to provide a quantitative chemical picture. The access to other properties, such as oscillator strengths, dipole moments, and analytical energy gradients, is also an asset if one wants to compare with experimental data. Let us not forget about the requirements of minimal user input and minimal chemical intuition (i.e., black box models are preferable) in order to minimize the potential bias brought by the user appreciation of the problem complexity. Finally, low computational scaling with respect to system size and small memory footprint cannot be disregarded. Although the simultaneous fulfillment of all these requirements seems elusive, it is useful to keep these criteria in mind. Table 1 is here for fulfill such a purpose. In this Table, we also provide the typical error bar associated with each of these methods. Table S1 of the supporting information reports additional details about (some of the) existing BSE and wave function theory benchmarks, whereas a review of TD-DFT benchmark studies can be found elsewhere. ${ }^{8}$ As can be seen in Table S1, the actual error bar obtained for a given method strongly depends on the actual type of excited states and compounds. Hence, the values listed in Table 1 should be viewed as "typical" errors for organic molecules, nothing more.

Before detailing some key past and present contributions aiming at obtaining highly accurate excitation energies, we start by giving a historical overview of the various excitedstate $a b$ initio methods that have emerged in the last fifty years. Interestingly, for pretty much every single method, the theory was derived much earlier than their actual implementation in electronic structure software packages and the same applies to the analytical gradients when available.

The first mainstream ab initio method for excited states was probably CIS (configuration interaction with singles) which has been around since the 1970's. ${ }^{9}$ CIS lacks electron correlation and therefore grossly overestimates excitation energies and wrongly orders excited states. It is not unusual to have errors of the order of $1 \mathrm{eV}$ which precludes the usage of CIS as a
Table 1: Formal computational scaling of various excited-state methods with respect to the number of one-electron basis functions $N$ and the accessibility of various key properties in popular computational software packages. For organic derivatives, the typical error range for single excitations is also provided as a qualitative indicator of the method accuracy.

\begin{tabular}{|c|c|c|c|c|}
\hline Method & $\begin{array}{l}\text { Formal } \\
\text { scaling }\end{array}$ & $\begin{array}{c}\text { Oscillator } \\
\text { strength }\end{array}$ & $\begin{array}{c}\text { Analytical } \\
\text { gradients }\end{array}$ & $\begin{array}{c}\text { Typical } \\
\text { error }(\mathrm{eV})\end{array}$ \\
\hline TD-DFT & $N^{4}$ & $\sqrt{ }$ & $\checkmark$ & $0.2-0.4^{a}$ \\
\hline BSE@GW & $N^{4}$ & $\checkmark$ & $x$ & $0.1-0.3^{b}$ \\
\hline CIS & $N^{5}$ & $\checkmark$ & $\checkmark$ & $\sim 1.0$ \\
\hline CIS(D) & $N^{5}$ & $x$ & $\checkmark$ & $0.2-0.3$ \\
\hline $\operatorname{ADC}(2)$ & $N^{5}$ & $\checkmark$ & $\checkmark$ & $0.1-0.2$ \\
\hline $\mathrm{CC} 2$ & $N^{5}$ & $\checkmark$ & $\checkmark$ & $0.1-0.2$ \\
\hline $\operatorname{ADC}(3)$ & $N^{6}$ & $\checkmark$ & $x$ & 0.2 \\
\hline EOM-CCSD & $N^{6}$ & $\checkmark$ & $\checkmark$ & $0.1-0.3$ \\
\hline CC3 & $N^{7}$ & $\checkmark$ & $x$ & $\sim 0.04$ \\
\hline EOM-CCSDT & $N^{8}$ & $x$ & $x$ & $\sim 0.03$ \\
\hline EOM-CCSDTQ & $N^{10}$ & $x$ & $x$ & $\sim 0.01$ \\
\hline CASPT2/NEVPT2 & $N !$ & $\checkmark$ & $\checkmark$ & $0.1-0.2$ \\
\hline SCI & $N !$ & $x$ & $x$ & $\sim 0.03$ \\
\hline FCI & $N !$ & $\checkmark$ & $\checkmark$ & 0.0 \\
\hline
\end{tabular}

${ }^{a}$ The error range is strongly functional and state dependent. The values reported here are for well-behaved cases; ${ }^{b}$ Typical error bar for singlet transitions. Larger errors are often observed for triplet excitations.

quantitative quantum chemistry method. Twenty years later, CIS(D) which adds a second-order perturbative correction to CIS was developed and implemented thanks to the efforts of Head-Gordon and coworkers. ${ }^{10,11}$ This second-order correction greatly reduces the magnitude of the error compared to CIS, with a typical error range of $0.2-0.3 \mathrm{eV}$.

In the early 1990's, the complete-active-space selfconsistent field (CASSCF) method ${ }^{12,13}$ and its second-order perturbation-corrected variant CASPT $2{ }^{14}$ (originally developed in Roos' group) became very popular. This was a real breakthrough. Although it took more than ten years to obtain analytical gradients, ${ }^{15}$ CASPT2 was probably the first method that could provide quantitative results for molecular excited states of genuine photochemical interest. ${ }^{16}$ Nonetheless, it is of common knowledge that CASPT2 has the clear tendency of underestimating vertical excitation energies in organic molecules. Driven by Angeli and Malrieu, ${ }^{17}$ the creation of the second-order $n$-electron valence state perturbation theory (NEVPT2) method several years later was able to cure some of the main theoretical deficiencies of CASPT2. For example, NEVPT2 is known to be intruder state free and size consistent. The limited applicability of these multiconfigurational methods is mainly due to the need of carefully defining an active space based on the desired transition(s) in order to obtain meaningful results, as well as their factorial computational growth with the number of active electrons 
and orbitals. With a typical minimal valence active space tailored for the desired transitions, the usual error with CASPT2 or NEVPT2 calculations is $0.1-0.2 \mathrm{eV}$, with the additional complication of the possible IPEA correction for the former method. ${ }^{18} \mathrm{We}$ also point out that some emergent approaches, like DMRG (density matrix renormalization group), ${ }^{19}$ offer a new path for the development of these multiconfigurational methods.

The advent of time-dependent density-functional theory (TD-DFT) $^{20,21}$ was a significant step for the community as TD-DFT was able to provide accurate excitation energies at a much lower cost than its predecessors in a black-box way. For low-lying valence excited states, TD-DFT calculations relying on hybrid exchange-correlation functionals have a typical error of $0.2-0.4 \mathrm{eV}$. However, a large number of shortcomings were quickly discovered. ${ }^{1,22-27}$ In the present context, one of the most annoying feature of TD-DFT — in its most standard (adiabatic) approximation - is its inability to describe, even qualitatively, charge-transfer states, ${ }^{23,24}$ Rydberg states, ${ }^{22}$ and double excitations. ${ }^{25-27}$ These issues, as well as other well-documented shortcomings of DFT and TD-DFT, are related to the so-called delocalization error. ${ }^{28}$ One closely related issue is the selection of the exchange-correlation functional from an ever-growing zoo of functionals and the variation of the excitation energies that one can observe with different functionals. ${ }^{29,30}$ More specifically, despite the development of new, more robust approaches (including the so-called range-separated ${ }^{31-34}$ and double ${ }^{35-37}$ hybrids), it is still difficult (not to say impossible) to select a functional adequate for all families of transitions. ${ }^{8}$ Moreover, the difficulty of making TD-DFT systematically improvable obviously hampers its applicability. Despite all of this, TD-DFT remains nowadays the most employed excited-state method in the electronic structure community (and beyond).

Thanks to the development of coupled cluster (CC) response theory, ${ }^{38}$ and the growth of computational resources, equation-of-motion coupled cluster with singles and doubles (EOM-CCSD) ${ }^{39}$ became mainstream in the 2000's. EOMCCSD gradients were also quickly available. ${ }^{40}$ With EOMCCSD, it is not unusual to have errors as small as $0.1 \mathrm{eV}$ for small compounds and generally $0.2 \mathrm{eV}$ for larger ones, with a typical overestimation of the vertical transition energies. Its third-order version, EOM-CCSDT, was also implemented and provides, at a significantly higher cost, high accuracy for single excitations. ${ }^{41}$ Although extremely expensive and tedious to implement, higher orders are also technically possible for small systems thanks to automatically generated code. ${ }^{42,43}$ For the sake of brevity, we drop the EOM acronym in the rest of this Perspective keeping in mind that these CC methods are applied to excited states in the present context.

The original CC family of methods was quickly completed by an approximated and computationally lighter family with, in front line, the second-order CC2 model $^{44}$ and its thirdorder extension, CC $3 .{ }^{45}$ As a $N^{7}$ method (where $N$ is the number of basis functions), CC3 has a particularly interesting accuracy/cost ratio with errors usually below the chemical accuracy threshold. ${ }^{46-49}$ The series CC2, CCSD, CC3, CCSDT defines a hierarchy of models with $N^{5}, N^{6}, N^{7}$ and $N^{8}$ scaling, respectively. It is also noteworthy that CCSDT and CC3 are also able to detect the presence of double excitations, a feature that is absent from both CCSD and CC2. ${ }^{50}$

It is also important to mention the recent rejuvenation of the second- and third-order algebraic diagrammatic construction $\left[\mathrm{ADC}(2)^{51}\right.$ and $\left.\mathrm{ADC}(3)^{52,53}\right]$ methods that scale as $N^{5}$ and $N^{6}$, respectively. These methods are related to the older second- and third-order polarization propagator approaches (SOPPA and TOPPA). ${ }^{54,55}$ This renaissance was certainly initiated by the enormous amount of work invested by Dreuw's group in order to provide a fast and efficient implementation of these methods, ${ }^{56}$ including the analytical gradients, ${ }^{57}$ as well as other interesting variants. ${ }^{56,58}$ These Green's function one-electron propagator techniques indeed represent valuable alternatives thanks to their reduced cost compared to their $\mathrm{CC}$ equivalents. In that regard, $\mathrm{ADC}(2)$ is particularly attractive with an error around $0.1-0.2 \mathrm{eV}$. However, we have recently observed that $\operatorname{ADC}(3)$ generally overcorrects the ADC(2) excitation energies and is significantly less accurate than CC3. ${ }^{47,59-61}$

Finally, let us mention the many-body Green's function Bethe-Salpeter equation (BSE) formalism ${ }^{62}$ (which is usually performed on top of a $G W$ calculation). ${ }^{63} \mathrm{BSE}$ has gained momentum in the past few years and is a serious candidate as a computationally inexpensive electronic structure theory method that can effectively model excited states with a typical error of $0.1-0.3 \mathrm{eV}$, as well as some related properties. $^{64,65}$ One of the main advantage of BSE compared to TD-DFT (with a similar computational cost) is that it allows a faithful description of charge-transfer states and, when performed on top of a (partially) self-consistently $G W$ calculation, BSE@ $G W$ has been shown to be weakly dependent on its starting point (i.e., on the functional selected for the underlying DFT calculation). ${ }^{66,67}$ However, due to the adiabatic (i.e., static) approximation, doubly excited states are completely absent from the BSE spectrum.

In the past five years, ${ }^{68,69}$ we have witnessed a resurgence of the so-called selected CI (SCI) methods ${ }^{70-72}$ thanks to the development and implementation of new, fast, and efficient algorithms to select cleverly determinants in the full CI (FCI) space (see Refs. 73,74 and references therein). SCI methods rely on the same principle as the usual CI approach, except that determinants are not chosen a priori based on occupation or excitation criteria but selected among the entire set of determinants based on their estimated contribution to the FCI wave function or energy. Indeed, it has been noticed long ago that, even inside a predefined subspace of determinants, only a small number of them significantly contributes. The main advantage of SCI methods is that no a priori assumption is made on the type of electron correlation. Therefore, at the price of a brute force calculation, a SCI calculation is not, or at least less, biased by the user appreciation of the problem's complexity. One of the strength of one of the implementation, based on the CIPSI (configuration interaction using a perturbative selection made iteratively) algorithm developed by Huron, Rancurel, and Malrieu ${ }^{72}$ is its parallel efficiency which makes possible to run on thousands of CPU cores. ${ }^{74}$ Thanks to these tremendous features, SCI methods deliver near FCI quality excitation energies for both singly and doubly excited states, ${ }^{47,50,75,76}$ with an error of roughly $0.03 \mathrm{eV}$, 
mostly originating from the extrapolation procedure. ${ }^{74}$ However, although the "exponential wall" is pushed back, this type of method is only applicable to molecules with a small number of heavy atoms and/or relatively compact basis sets.

For someone who has never worked with SCI methods, it might be surprising to see that one is able to compute near-FCI excitation energies for molecules as big as benzene. ${ }^{50,60,76}$ This is mainly due to some specific choices in terms of implementation as explained below. Indeed, to keep up with Moore's "Law" in the early 2000's, the processor designers had no other choice than to propose multi-core chips to avoid an explosion of the energy requirements. Increasing the number of floating-point operations per second by doubling the number of CPU cores only requires to double the required energy, while doubling the frequency multiplies the required energy by a factor of $\sim 8$. This bifurcation in hardware design implied a change of paradigm ${ }^{77}$ in the implementation and design of computational algorithms. A large degree of parallelism is now required to benefit from a significant acceleration. Fifteen years later, the community has made a significant effort to redesign the methods with parallel-friendly algorithms. ${ }^{78-83}$ In particular, the change of paradigm to reach FCI accuracy with SCI methods came from the use of determinant-driven algorithms which were considered for long as inefficient with respect to integraldriven algorithms. The first important element making these algorithms efficient is the introduction of new bit manipulation instructions (BMI) in the hardware that enable an extremely fast evaluation of Slater-Condon rules ${ }^{84}$ for the direct calculation of the Hamiltonian matrix elements over arbitrary determinants. Then massive parallelism can be harnessed to compute the second-order perturbative correction with semi-stochatic algorithms, ${ }^{80,85}$ and perform the sparse matrix multiplications required in Davidson's algorithm to find the eigenvectors associated with the lowest eigenvalues. Block-Davidson methods can require a large amount of memory, and the recent introduction of byte-addressable non-volatile memory as a new tier in the memory hierarchy ${ }^{86}$ will enable SCI calculations on larger molecules. The next generation of supercomputers is going to generalize the presence of accelerators (graphical processing units, GPUs), leading to a new software crisis. Fortunately, some authors have already prepared this transition. ${ }^{87-91}$

In summary, each method has its own strengths and weaknesses, and none of them is able to provide accurate, balanced, and reliable excitation energies for all classes of electronic excited states at an affordable cost.

Although sometimes decried, benchmark sets of molecules and their corresponding reference data are essential for the validation of existing theoretical models and to bring to light and subsequently understand their strengths and, more importantly, their limitations. These sets have started to emerge at the end of the 1990's for ground-state properties with the acclaimed G2 test set designed by the Pople group. ${ }^{92}$ For excited states, things started moving a little later but some major contributions were able to put things back on track.

One of these major contributions was provided by the group of Walter Thiel ${ }^{93-97}$ with the introduction of the so-called Thiel (or Mülheim) set of excitation energies. ${ }^{93}$ For the first time, this set was large, diverse, consistent, and accurate enough to be used as a proper benchmarking set for excitedstate methods. More specifically, it gathers a large number of excitation energies consisting of 28 medium-size organic molecules with a total of 223 valence excited states (152 singlet and 71 triplet states) for which theoretical best estimates (TBEs) were defined. In their first study Thiel and collaborators performed CC2, CCSD, CC3 and CASPT2 calculations (with the TZVP basis) in order to provide (based on additional high-quality literature data) TBEs for these transitions. Their main conclusion was that " $C C 3$ and CASPT2 excitation energies are in excellent agreement for states which are dominated by single excitations". These TBEs were quickly refined with the larger aug-cc-pVTZ basis set, ${ }^{96}$ highlighting the importance of diffuse functions. As a direct evidence of the actual value of reference data, these TBEs were quickly picked up to benchmark various computationally effective methods from semi-empirical to state-of-the-art $a b$ initio methods (see the Introduction of Ref. 47 and references therein).

Theoretical improvements of Thiel's set were slow but steady, highlighting further its quality. ${ }^{53,98-100}$ In 2013, Watson et al. ${ }^{98}$ computed CCSDT-3/TZVP (an iterative approximation of the triples of CCSDT ${ }^{101}$ ) excitation energies for the Thiel set. Their quality were very similar to the CC3 values reported in Ref. 95 and the authors could not appreciate which model was the most accurate. Similarly, Dreuw and coworkers performed ADC(3) calculations on Thiel's set and arrived at the same kind of conclusion: 53 "based on the quality of the existing benchmark set it is practically not possible to judge whether ADC(3) or CC3 is more accurate”. These two studies clearly demonstrate and motivate the need for higher accuracy benchmark excited-states energies.

Recently, we made, what we think, is a significant contribution to this quest for highly accurate vertical excitation energies. ${ }^{47}$ More specifically, we studied 18 small molecules with sizes ranging from one to three non-hydrogen atoms. For such systems, using a combination of high-order CC methods, SCI calculations and large diffuse basis sets, we were able to compute a list of 110 highly accurate vertical excitation energies for excited states of various natures (valence, Rydberg, $n \rightarrow \pi^{*}, \pi \rightarrow \pi^{*}$, singlet, triplet and doubly excited) based on CC3/aug-cc-pVTZ geometries. In the following, we label this set of TBEs as QUEST\#1. Importantly, it allowed us to benchmark a series of popular excited-state wave function methods partially or fully accounting for double and triple excitations (see Fig. 1): CIS(D), CC2, CCSD, STEOMCCSD ${ }^{102} \operatorname{CCSDR}(3),{ }^{103} \mathrm{CCSDT}-3,{ }^{101} \mathrm{CC} 3, \mathrm{ADC}(2)$, and $\mathrm{ADC}(3)$. Our main conclusion was that CC3 is extremely accurate (with a mean absolute error of only $\sim 0.03 \mathrm{eV}$ ), and that, although slightly less accurate than CC3, CCSDT-3 could be used as a reliable reference for benchmark studies. Quite surprisingly, ADC(3) was found to have a clear tendency to overcorrect its second-order version $\mathrm{ADC}(2)$. The mean absolute errors (MAEs) obtained for this set can be found in Fig. 1.

In a second study, ${ }^{50}$ using a similar combination of theoretical models (but mostly extrapolated SCI energies), we provided accurate reference excitation energies for transitions involving a substantial amount of double excitations 
QUEST\#1 test set (1 to 3 non-hydrogen atoms)
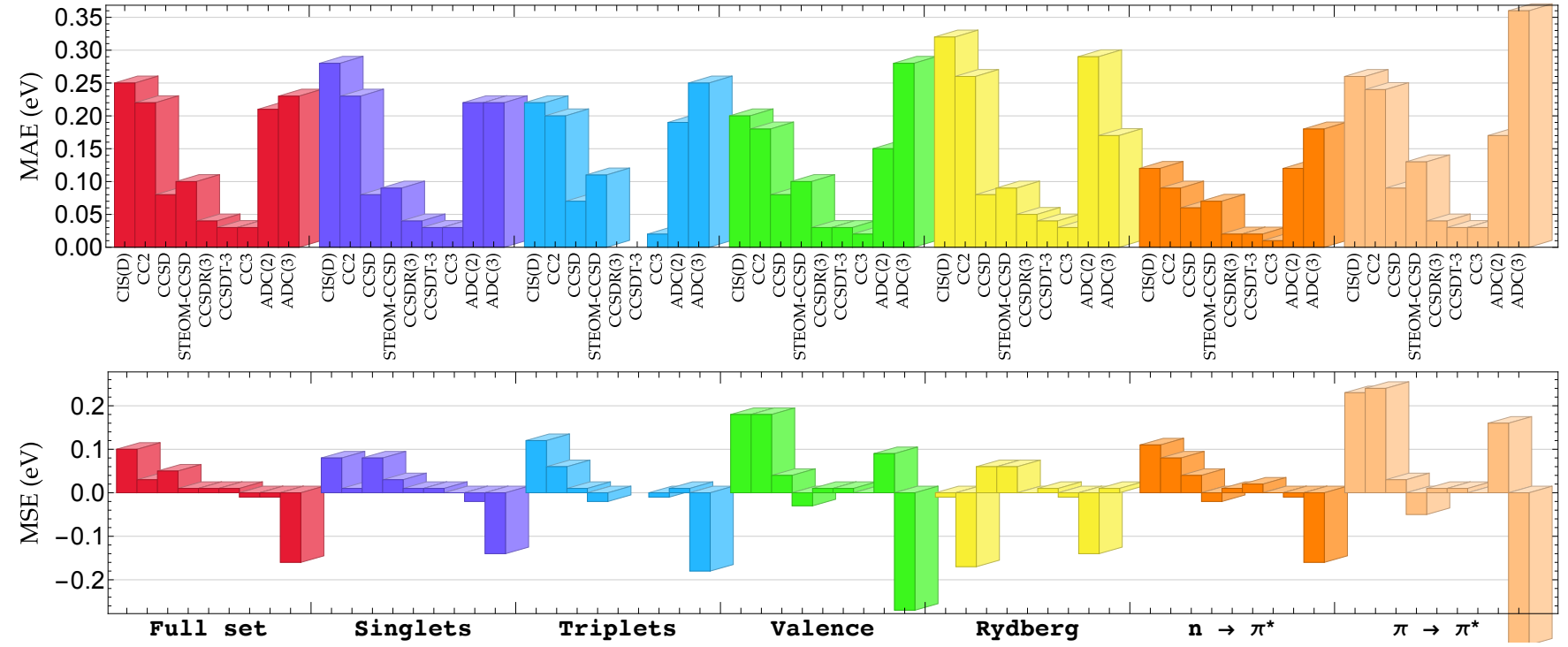

Figure 1: Mean absolute error (MAE, top) and mean signed error (MSE, bottom) with respect to the TBE/aug-cc-pVTZ values from the QUEST\#1 set (as described in Ref. 47) for various methods and types of excited states. The corresponding graph for the maximum positive and negative errors can be found in the supporting information.

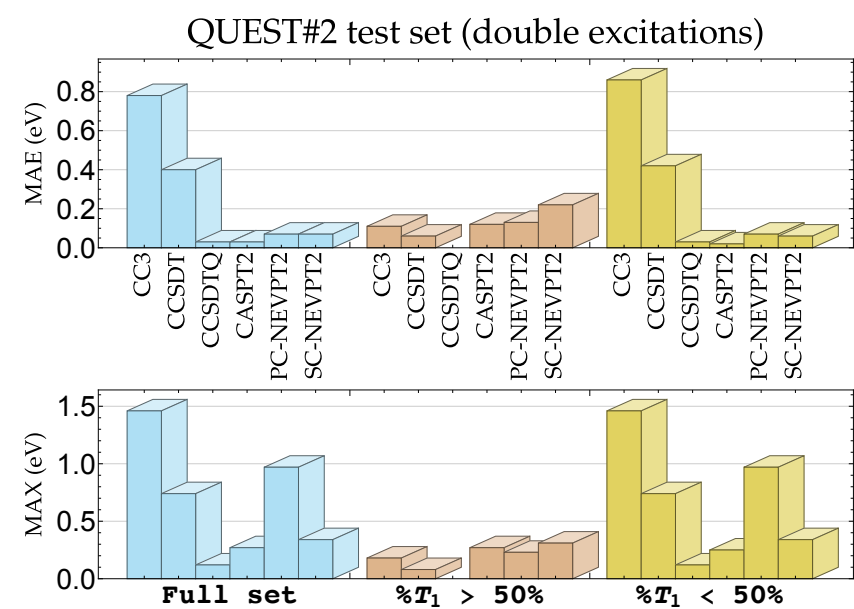

Figure 2: Mean absolute error (MAE, top) and maximum absolute error (MAX, bottom) with respect to $\mathrm{FCl}$ excitation energies for the doubly excited states reported in Ref. 50 for various methods taking into account at least triple excitations. $\% T_{1}$ corresponds to single excitation percentage in the transition calculated at the CC3 level. For this particular set and methods, the mean signed error is equal to the MAE.

using a series of increasingly large diffuse-containing atomic basis sets (up to aug-cc-pVQZ when technically feasible). This set gathers 20 vertical transitions from 14 small- and medium-sized molecules, a set we label as QUEST\#2 in the remaining of this Perspective. An important addition to this second study was the inclusion of various flavors of multiconfigurational methods (CASSCF, CASPT2, and NEVPT2) in addition to high-order $\mathrm{CC}$ methods including, at least, perturbative triples (see Fig. 2). Our results clearly evidence that the error in CC methods is intimately related to the amount of double-excitation character in the vertical transition. For "pure" double excitations (i.e., for transitions which do not mix with single excitations), the error in CC3 and CCSDT can easily reach 1 and $0.5 \mathrm{eV}$, respectively, while it goes down to a few tenths of an electronvolt for more common transitions (such as in trans-butadiene and benzene) involving a significant amount of singles. ${ }^{104-106}$ The quality of the excitation energies obtained with multiconfigurational methods was harder to predict as the overall accuracy of these methods is highly dependent on both the system and the selected active space. Nevertheless, CASPT2 and NEVPT2 were found to be more accurate for transition with a small percentage of single excitations (error usually below $0.1 \mathrm{eV}$ ) than for excitations dominated by single excitations where the error is closer from 0.1-0.2 eV (see Fig. 2).

In our latest study, ${ }^{60}$ in order to provide more general conclusions, we generated highly accurate vertical transition energies for larger compounds with a set composed by 27 organic molecules encompassing from four to six nonhydrogen atoms for a total of 223 vertical transition energies of various natures. This set, labeled as QUEST\#3 and still based on CC3/aug-cc-pVTZ geometries, is constituted by a reasonably good balance of singlet, triplet, valence, and Rydberg states. To obtain this new, larger set of TBEs, we employed CC methods up to the highest possible order (CC3, CCSDT, and CCSDTQ), very large SCI calculations (with up to hundred million determinants), as well as the most robust multiconfigurational method, NEVPT2. Each approach was applied in combination with diffuse-containing atomic basis sets. For all the transitions of the QUEST\#3 set, we reported at least CCSDT/aug-cc-pVTZ (sometimes with basis set extrapolation) and CC3/aug-cc-pVQZ transition energies as well as CC3/aug-cc-pVTZ oscillator strengths for each dipole-allowed transition. Pursuing our previous benchmarking efforts, ${ }^{47,50}$ we confirmed that CC 3 almost systematically delivers transition energies in agreement with higher-level 
QUEST\#3 test set (4 to 6 non-hydrogen atoms)
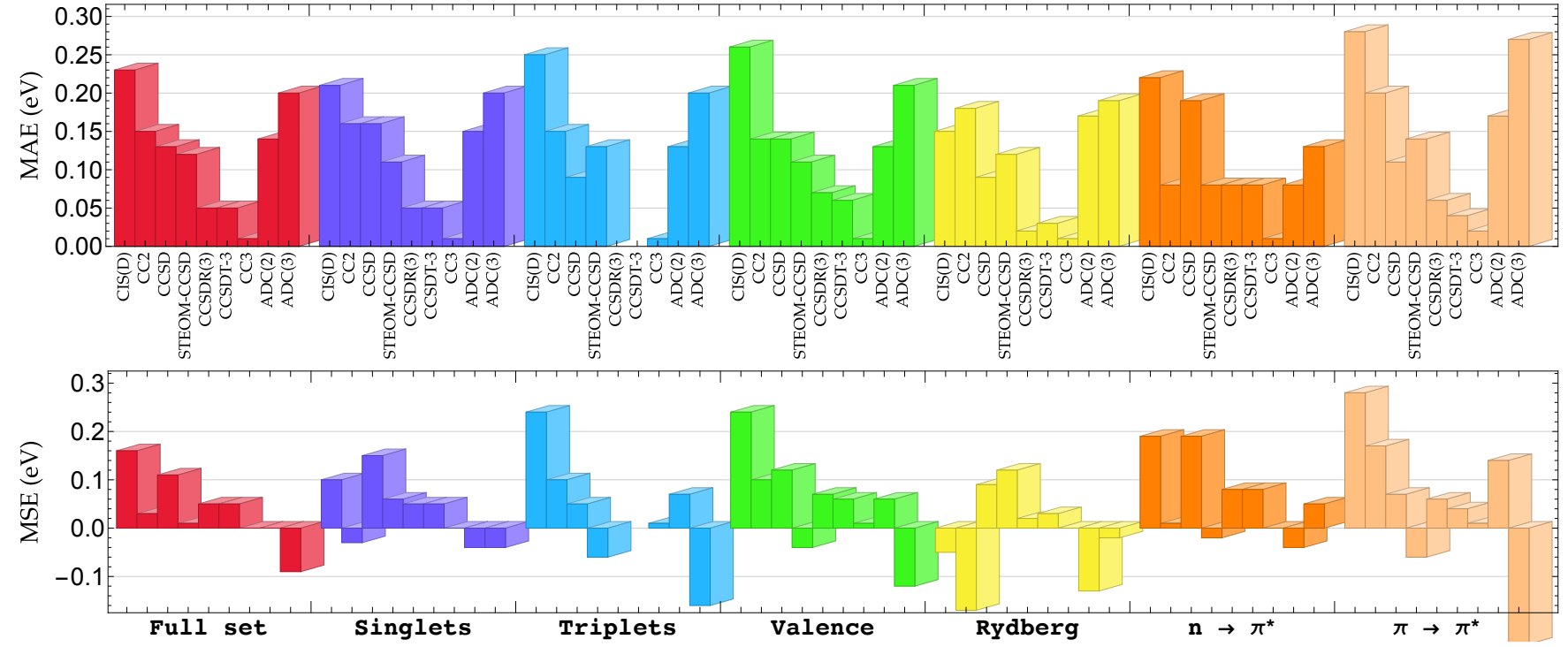

Figure 3: Mean absolute error (MAE, top) and mean signed error (MSE, bottom) with respect to the TBE/aug-cc-pVTZ values from the QUEST\#3 set (as described in Ref. 60) for various methods and types of excited states. The corresponding graph for the maximum positive and negative errors can be found in the supporting information.

theoretical models $( \pm 0.04 \mathrm{eV})$ except for transitions presenting a dominant double excitation character (see Fig. 3). This settles down, at least for now, the debate by demonstrating the superiority of $\mathrm{CC} 3$ (in terms of accuracy) compared to methods like CCSDT-3 or ADC(3), see Fig. 3. Moreover, thanks to the exhaustive and detailed comparisons made in Ref. 60, we could safely conclude that CC 3 also regularly outperforms CASPT2 (which often underestimates excitation energies) and NEVPT2 (which typically overestimates excitation energies) as long as the corresponding transition does not show any strong multiple excitation character.

Our current efforts are now focussing on expanding and merging these sets to create an complete test set of highly accurate excitations energies. In particular, we are currently generating reference excitations energies for radicals as well as more "exotic" molecules containing heavier atoms (such as $\mathrm{Cl}, \mathrm{P}$, and $\mathrm{Si}$ ). The combination of these various sets would potentially create an ensemble of more than 400 vertical transition energies for small- and medium-size molecules based on accurate ground-state geometries. Such a set would likely be a valuable asset for the electronic structure community. It would likely stimulate further theoretical developments in excited-state methods and provide a fair ground for the assessments of the currently available and under development excited-state models.

Besides all the studies described above aiming at reaching chemically accurate vertical transition energies, it should be pointed out that an increasing amount of effort is currently devoted to the obtention of highly-trustable excited-state properties. This includes, first, 0-0 energies, ${ }^{4-7,35,46,48,49,107}$ which, as mentioned above, offer well-grounded comparisons with experiment. However, because $0-0$ energies are fairly insensitive to the underlying molecular geometries, ${ }^{5,49,107}$ they are not a good indicator of their overall quality. Consequently, one can find in the literature several sets of excited-state ge- ometries obtained at various levels of theory, ${ }^{108-112}$ some of them being determined using state-of-the-art models. ${ }^{109,112}$ There are also investigations of the accuracy of the nuclear gradients at the Franck-Condon point. ${ }^{13,114}$ The interested reader may find useful several investigations reporting sets of reference oscillator strengths. ${ }^{47,53,61,97,99}$ Up to now, these investigations focusing on geometries and oscillator strengths have been mostly based on theory-vs-theory comparisons. Indeed, while for small compounds (i.e., typically from dito tetra-atomic molecules), one can find very accurate experimental measurements (excited state dipole moments, oscillator strengths, vibrational frequencies, etc), these data are usually not accessible for larger compounds. Nevertheless, the emergence of X-ray free electron lasers might soon allow to obtain accurate experimental excited state densities and geometrical structures through diffraction experiments. Such new experimental developments will likely offer new opportunities for experiment-vs-theory comparisons going beyond standard energetics. Finally, more complex properties, such as two-photon cross-sections and vibrations, have been mostly determined at lower levels of theory, hinting at future studies on this particular subject.

As concluding remarks, we would like to highlight once again the major contribution brought by Roos' and Thiel's groups in an effort to define benchmark values for excited states. Following their footsteps, we have recently proposed a larger, even more accurate set of vertical transitions energies for various types of excited states (including double excitations). ${ }^{47,50,60}$ This was made possible thanks to a technological renaissance of SCI methods which can now routinely produce near-FCI excitation energies for small- and medium-size organic molecules. ${ }^{73,74,76}$ We hope that new technological advances will enable us to push further, in years to come, our quest to highly accurate excitation energies, and, importantly, of other excited-state properties. 
PFL would like to thank Peter Gill for useful discussions. He also acknowledges funding from the "Centre National de la Recherche Scientifique”. DJ acknowledges the Région des Pays de la Loire for financial support.

\section{REFERENCES}

(1) Dreuw, A.; Head-Gordon, M. Single-Reference $a b$ initio Methods for the Calculation of Excited States of Large Molecules. Chem. Rev. 2005, 105, 4009-4037.

(2) González, L.; Escudero, D.; Serrano-Andrès, L. Progress and Challenges in the Calculation of Electronic Excited States. ChemPhysChem 2012, 13, 2851.

(3) Ghosh, S.; Verma, P.; Cramer, C. J.; Gagliardi, L.; Truhlar, D. G. Combining Wave Function Methods with Density Functional Theory for Excited States. Chem. Rev. 2018, 118, 7249-7292.

(4) Dierksen, M.; Grimme, S. The Vibronic Structure of Electronic Absorption Spectra of Large Molecules: A Time-Dependent Density Functional Study on the Influence of Exact Hartree-Fock Exchange. J. Phys. Chem. A 2004, 108, 10225-10237.

(5) Winter, N. O. C.; Graf, N. K.; Leutwyler, S.; Hättig, C. Benchmarks for 0-0 Transitions of Aromatic Organic Molecules: DFT/B3LYP, ADC(2), CC2, SOS-CC2 and SCS-CC2 Compared to High-resolution GasPhase Data. Phys. Chem. Chem. Phys. 2013, 15, 66236630.

(6) Fang, C.; Oruganti, B.; Durbeej, B. How MethodDependent Are Calculated Differences Between Vertical, Adiabatic and 0-0 Excitation Energies? J. Phys. Chem. A 2014, 118, 4157-4171.

(7) Loos, P.-F.; Jacquemin, D. Evaluating 0-0 Energies with Theoretical Tools: a Short Review. ChemPhotoChem 2019, 3, 684-696.

(8) Laurent, A. D.; Jacquemin, D. TD-DFT Benchmarks: A Review. Int. J. Quantum Chem. 2013, 113, 20192039.

(9) Del Bene, J. E.; Ditchfield, R.; Pople, J. A. SelfConsistent Molecular Orbital Methods. X. Molecular Orbital Studies of Excited States with Minimal and Extended Basis Sets. J. Chem. Phys. 1971, 55, 2236.

(10) Head-Gordon, M.; Rico, R. J.; Oumi, M.; Lee, T. J. A Doubles Correction To Electronic Excited States From Configuration Interaction In The Space Of Single Substitutions. Chem. Phys. Lett. 1994, 219, 21.

(11) Ishikawa, N.; Head-Gordon, M. Analytical Gradient of the CIS(D) Perturbative Correction to SingleExcitation Configuration Interaction Excited States. Int. J. Quantum Chem. 1995, 56, 421-427.

(12) Roos, B. O.; Taylor, P. R.; Sigbahn, P. E. A Complete Active Space SCF Method (CASSCF) Using a Density Matrix Formulated Super-CI Approach. Chem. Phys. 1980, 48, 157-173.

(13) Andersson, K.; Malmqvist, P. A.; Roos, B. O.; Sadlej, A. J.; Wolinski, K. Second-Order Perturba- tion Theory With a CASSCF Reference Function. $J$. Phys. Chem. 1990, 94, 5483-5488.

(14) Andersson, K.; Malmqvist, P.-A.; Roos, B. O. SecondOrder Perturbation Theory With a Complete Active Space Self-Consistent Field Reference Function. $J$. Chem. Phys. 1992, 96, 1218-1226.

(15) Celani, P.; Werner, H.-J. Analytical Energy Gradients for Internally Contracted Second-Order Multireference Perturbation Theory. J. Chem. Phys. 2003, 119, 5044-5057.

(16) Roos, B. O.; Andersson, K.; Fulscher, M. P.; Malmqvist, P.-A.; Serrano-Andrés, L. In Multiconfigurational Perturbation Theory: Applications In Electronic Spectroscopy; Prigogine, I., Rice, S. A., Eds.; Adv. Chem. Phys.; Wiley, New York, 1996; Vol. XCIII; pp 219-331.

(17) Angeli, C.; Cimiraglia, R.; Evangelisti, S.; Leininger, T.; Malrieu, J.-P. Introduction of $N$ Electron Valence States for Multireference Perturbation Theory. J. Chem. Phys. 2001, 114, 10252-10264.

(18) Zobel, J. P.; Nogueira, J. J.; Gonzalez, L. The IPEA Dilemma in CASPT2. Chem. Sci. 2017, 8, 1482-1499.

(19) Baiardi, A.; Reiher, M. The Density Matrix Renormalization Group in Chemistry and Molecular Physics: Recent Developments and New Challenges. 2019; arXiv, 1910.00137.

(20) Runge, E.; Gross, E. K. U. Density-Functional Theory for Time-Dependent Systems. Phys. Rev. Lett. 1984, 52, 997-1000.

(21) Casida, M. E. In Time-Dependent Density-Functional Response Theory for Molecules; Chong, D. P., Ed.; Recent Advances in Density Functional Methods; World Scientific: Singapore, 1995; Vol. 1; pp 155192.

(22) Tozer, D. J.; Handy, N. C. Improving Virtual Kohn-Sham Orbitals and Eigenvalues: Application to Excitation Energies and Static Polarizabilities. $J$. Chem. Phys. 1998, 109, 10180-10189.

(23) Tozer, D. J.; Amos, R. D.; Handy, N. C.; Roos, B. O.; Serrano-Andrés, L. Does Density Functional Theory Contribute to the Understanding of Excited States of Unsaturated Organic Compounds? Mol. Phys. 1999, 97, 859-868.

(24) Dreuw, A.; Head-Gordon, M. Failure of TimeDependent Density Functional Theory for Long-Range Charge-Transfer Excited States: the Zincbacteriochlorin-Bacteriochlorin and Bacteriochlorophyll-Spheroidene Complexes. $J$. Am. Chem. Soc. 2004, 126, 4007-4016.

(25) Maitra, N. T.; Zhang, F.; Cave, R. J.; Burke, K. Double Excitations within Time-Dependent Density Functional Theory Linear Response. J. Chem. Phys. 2004, 120, 5932-5937.

(26) Levine, B. G.; Ko, C.; Quenneville, J.; Martínez, T. J. Conical Intersections and Double Excitations in TimeDependent Density Functional Theory. Mol. Phys. 2006, 104, 1039-1051.

(27) Elliott, P.; Goldson, S.; Canahui, C.; Maitra, N. T. 
Perspectives on double-excitations in TDDFT. Chem. Phys. 2011, 391, 110-119.

(28) Autschbach, J.; Srebro, M. Delocalization Error and "Functional Tuning" in Kohn-Sham Calculations of Molecular Properties. Acc. Chem. Res. 2014, 47, 2592-2602.

(29) Goerigk, L.; Mehta, N. A Trip To The Density Functional Theory Zoo: Warnings And Recommendations For The User. Aus. J. Chem. 2019, 72, 563-573.

(30) Suellen, C.; Garcia Freitas, R.; Loos, P.-F.; Jacquemin, D. Cross Comparisons Between Experiment, TD-DFT, CC, and ADC for Transition Energies. J. Chem. Theory Comput. 2019, 15, 4581-4590.

(31) Savin, A. In Recent Developments and Applications of Modern Density Functional Theory; Seminario, J. M., Ed.; Elsevier: Amsterdam, 1996; Chapter 9, pp $327-$ 354.

(32) Iikura, H.; Tsuneda, T.; Yanai, T.; Hirao, K. A LongRange Correction Scheme for Generalized-GradientApproximation Exchange Functionals. J. Chem. Phys. 2001, 115, 3540-3544.

(33) Yanai, T.; Tew, D. P.; Handy, N. C. A New Hybrid Exchange-Correlation Functional Using the CoulombAttenuating Method (CAM-B3LYP). Chem. Phys. Lett. 2004, 393, 51-56.

(34) Vydrov, O. A.; Scuseria, G. E. Assessment of a longrange corrected hybrid functional. J. Chem. Phys. 2006, 125, 234109.

(35) Goerigk, L.; Grimme, S. Assessment of TD-DFT Methods and of Various Spin Scaled CIS $_{n}$ D and CC2 Versions for the Treatment of Low-Lying Valence Excitations of Large Organic Dyes. J. Chem. Phys. 2010, 132, 184103.

(36) Brémond, E.; Ciofini, I.; Sancho-García, J. C.; Adamo, C. Nonempirical Double-Hybrid Functionals: An Effective Tool for Chemists. Acc. Chem. Res. 2016, 49, 1503-1513.

(37) Schwabe, T.; Goerigk, L. Time-Dependent DoubleHybrid Density Functionals with Spin-Component and Spin-Opposite Scaling. J. Chem. Theory Comput. 2017, 13, 4307-4323.

(38) Koch, H.; Jørgensen, P. Coupled Cluster Response Functions. J. Chem. Phys. 1990, 93, 3333-3344.

(39) Stanton, J. F.; Bartlett, R. J. The Equation of Motion Coupled-Cluster Method - A Systematic Biorthogonal Approach to Molecular Excitation Energies, Transition-Probabilities, and Excited-State Properties. J. Chem. Phys. 1993, 98, 7029-7039.

(40) Stanton, J. F.; Gauss, J. Analytic Energy Derivatives for the Equation-of-Motion Coupled-Cluster Method: Algebraic Expressions, Implementation and Application to the $S_{1}$ State of HFCO. Theor. Chim. Acta 1995, 91, 267-289.

(41) Kucharski, S. A.; Włoch, M.; Musiał, M.; Bartlett, R. J. Coupled-Cluster Theory for Excited Electronic States: The Full Equation-Of-Motion Coupled-Cluster Single, Double, and Triple Excitation Method. J. Chem. Phys. 2001, 115, 8263-8266.
(42) Kucharski, S. A.; Bartlett, R. J. Recursive Intermediate Factorization and Complete Computational Linearization of the Coupled-Cluster Single, Double, Triple, and Quadruple Excitation Equations. Theor. Chim. Acta 1991, 80, 387-405.

(43) Hirata, S. Higher-Order Equation-of-Motion Coupled-Cluster Methods. J. Chem. Phys. 2004, 121, 51-59.

(44) Christiansen, O.; Koch, H.; Jørgensen, P. The SecondOrder Approximate Coupled Cluster Singles and Doubles Model CC2. Chem. Phys. Lett. 1995, 243, 409418.

(45) Christiansen, O.; Koch, H.; Jørgensen, P. Response Functions in the CC3 Iterative Triple Excitation Model. J. Chem. Phys. 1995, 103, 7429-7441.

(46) Hättig, C. In Response Theory and Molecular Properties (A Tribute to Jan Linderberg and Poul Jørgensen); Jensen, H. A., Ed.; Advances in Quantum Chemistry; Academic Press, 2005; Vol. 50; pp 37-60.

(47) Loos, P.-F.; Scemama, A.; Blondel, A.; Garniron, Y.; Caffarel, M.; Jacquemin, D. A Mountaineering Strategy to Excited States: Highly-Accurate Reference Energies and Benchmarks. J. Chem. Theory Comput. 2018, 14, 4360-4379.

(48) Loos, P.-F.; Galland, N.; Jacquemin, D. Theoretical 00 Energies with Chemical Accuracy. J. Phys. Chem. Lett. 2018, 9, 4646-4651.

(49) Loos, P.-F.; Jacquemin, D. Chemically Accurate 0-0 Energies with not-so-Accurate Excited State Geometries. J. Chem. Theory Comput. 2019, 15, 2481-2491.

(50) Loos, P.-F.; Boggio-Pasqua, M.; Scemama, A.; Caffarel, M.; Jacquemin, D. Reference Energies for Double Excitations. J. Chem. Theory Comput. 2019, 15, 1939-1956.

(51) Schirmer, J. Beyond The Random-Phase Approximation: a New Approximation Scheme For The Polarization Propagator. Phys. Rev. A 1982, 26, 2395-2416.

(52) Trofimov, A. B.; Stelter, G.; Schirmer, J. A Consistent Third-Order Propagator Method For Electronic Excitation. J. Chem. Phys. 1999, 111, 9982-9999.

(53) Harbach, P. H. P.; Wormit, M.; Dreuw, A. The Third-Order Algebraic Diagrammatic Construction Method (ADC(3)) for the Polarization Propagator for Closed-Shell Molecules: Efficient Implementation and Benchmarking. J. Chem. Phys. 2014, 141, 064113.

(54) Oddershede, J. Polarization Propagator Calculations. Adv. Quantum Chem. 1978, 11, 275-352.

(55) Packer, M. J.; Dalskov, E. K.; Enevoldsen, T.; Jensen, H. J. A.; Oddershede, J. A new Implementation of the Second-Order Polarization Propagator Approximation (SOPPA): The Excitation Spectra of Benzene and Naphthalene. J. Chem. Phys. 1996, 105, 5886.

(56) Dreuw, A.; Wormit, M. The Algebraic Diagrammatic Construction Scheme for the Polarization Propagator for the Calculation of Excited States. WIREs Comput. Mol. Sci. 2015, 5, 82-95. 
(57) Rehn, D. R.; Dreuw, A. Analytic Nuclear Gradients of the Algebraic-Diagrammatic Construction Scheme for the Polarization Propagator up to Third Order of Perturbation Theory. J. Chem. Phys. 2019, 150, 174110.

(58) Hodecker, M.; Dempwolff, A. L.; Rehn, D. R.; Dreuw, A. Algebraic-diagrammatic construction scheme for the polarization propagator including ground-state coupled-cluster amplitudes. I. Excitation energies. J. Chem. Phys. 2019, 150, 174104.

(59) Trofimov, A. B.; Stelter, G.; Schirmer, J. Electron Excitation Energies Using a Consistent Third-Order Propagator Approach: Comparison with Full Configuration Interaction and Coupled Cluster Results. $J$. Chem. Phys. 2002, 117, 6402-6410.

(60) Loos, P. F.; Lipparini, F.; Boggio-Pasqua, M.; Scemama, A.; Jacquemin, D. A Mountaineering Strategy to Excited States: Highly-Accurate Energies and Benchmarks for Medium Size Molecules. J. Chem. Theory Comput. 2020, submitted.

(61) Loos, P. F.; Jacquemin, D. Is ADC(3) as Accurate as CC3 for Valence and Rydberg Transition Energies? $J$. Phys. Chem. Lett. 2020, 11, 974-980.

(62) Strinati, G. Application of the Green's Functions Method to the Study of the Optical Properties of Semiconductors. Riv. Nuovo Cimento 1988, 11, 1-86.

(63) Hedin, L. New Method for Calculating the OneParticle Green's Function with Application to the Electron-Gas Problem. Phys. Rev. 1965, 139, A796.

(64) Jacquemin, D.; Duchemin, I.; Blase, X. Is the BetheSalpeter Formalism Accurate for Excitation Energies? Comparisons with TD-DFT, CASPT2, and EOMCCSD. J. Phys. Chem. Lett. 2017, 8, 1524-1529.

(65) Blase, X.; Duchemin, I.; Jacquemin, D. The BetheSalpeter Equation in Chemistry: Relations with TDDFT, Applications and Challenges. Chem. Soc. Rev. 2018, 47, 1022-1043.

(66) Jacquemin, D.; Duchemin, I.; Blase, X. Assessment of the Convergence of Partially Self-Consistent BSE/GW Calculations. Mol. Phys. 2016, 114, 957-967.

(67) Gui, X.; Holzer, C.; Klopper, W. Accuracy Assessment of $G W$ Starting Points for Calculating Molecular Excitation Energies Using the Bethe-Salpeter Formalism. J. Chem. Theory Comput. 2018, 14, 2127-2136.

(68) Giner, E.; Scemama, A.; Caffarel, M. Using Perturbatively Selected Configuration Interaction in Quantum Monte Carlo Calculations. Can. J. Chem. 2013, 91, 879-885.

(69) Giner, E.; Scemama, A.; Caffarel, M. Fixed-Node Diffusion Monte Carlo Potential Energy Curve of the Fluorine Molecule $\mathrm{F}_{2}$ Using Selected Configuration Interaction Trial Wavefunctions. J. Chem. Phys. 2015, 142, 044115.

(70) Bender, C. F.; Davidson, E. R. Studies in Configuration Interaction: The First-Row Diatomic Hydrides. Phys. Rev. 1969, 183, 23-30.

(71) Whitten, J. L.; Hackmeyer, M. Configuration Interaction Studies of Ground and Excited States of Polyatomic Molecules. I. The CI Formulation and Studies of Formaldehyde. J. Chem. Phys. 1969, 51, 5584 5596.

(72) Huron, B.; Malrieu, J. P.; Rancurel, P. Iterative Perturbation Calculations of Ground and Excited State Energies from Multiconfigurational Zeroth-Order Wavefunctions. J. Chem. Phys. 1973, 58, 5745-5759.

(73) Garniron, Y.; Scemama, A.; Giner, E.; Caffarel, M.; Loos, P.-F. Selected Configuration Interaction Dressed by Perturbation. J. Chem. Phys. 2018, 149, 064103.

(74) Garniron, Y.; Gasperich, K.; Applencourt, T.; Benali, A.; Ferté, A.; Paquier, J.; Pradines, B.; Assaraf, R.; Reinhardt, P.; Toulouse, J. et al. Quantum Package 2.0: A Open-Source Determinant-Driven Suite Of Programs. J. Chem. Theory Comput. 2019, 15, 3591-3609.

(75) Holmes, A. A.; Umrigar, C. J.; Sharma, S. Excited States Using Semistochastic Heat-Bath Configuration Interaction. J. Chem. Phys. 2017, 147, 164111.

(76) Chien, A. D.; Holmes, A. A.; Otten, M.; Umrigar, C. J.; Sharma, S.; Zimmerman, P. M. Excited States of Methylene, Polyenes, and Ozone from HeatBath Configuration Interaction. J. Phys. Chem. A 2018, 122, 2714-2722.

(77) Sutter, H.; Larus, J. Software and the Concurrency Revolution. Queue 2005, 3, 54-62.

(78) Valiev, M.; Bylaska, E. J.; Govind, N.; Kowalski, K.; Straatsma, T. P.; Van Dam, H. J. J.; Wang, D.; Nieplocha, J.; Apra, E.; Windus, T. L. et al. NWChem: A Comprehensive and Scalable Open-Source Solution for Large Scale Molecular Simulations. Comput. Phys. Commun. 2010, 181, 1477-1489.

(79) Cleland, D.; Booth, G. H.; Alavi, A. Communications: Survival of the Fittest: Accelerating Convergence in Full Configuration-Interaction Quantum Monte Carlo. J. Chem. Phys. 2010, 132, 041103.

(80) Garniron, Y.; Scemama, A.; Loos, P.-F.; Caffarel, M. Hybrid Stochastic-Deterministic Calculation of the Second-Order Perturbative Contribution of Multireference Perturbation Theory. J. Chem. Phys. 2017, 147, 034101.

(81) Peng, C.; Calvin, J. A.; Pavošević, F.; Zhang, J.; Valeev, E. F. Massively Parallel Implementation of Explicitly Correlated Coupled-Cluster Singles and Doubles Using TiledArray Framework. J. Phys. Chem. A 2016, 120, 10231-10244.

(82) Kristensen, K.; Kjærgaard, T.; Høyvik, I.-M.; Ettenhuber, P.; Jørgensen, P.; Jansik, B.; Reine, S.; Jakowski, J. The Divide-Expand-Consolidate MP2 Scheme Goes Massively Parallel. Mol. Phys. 2013, 111, 1196-1210.

(83) Scemama, A.; Caffarel, M.; Oseret, E.; Jalby, W. Quantum Monte Carlo for Large Chemical Systems: Implementing Efficient Strategies for Petascale Platforms and Beyond. J. Comput. Chem. 2013, 34, 938951.

(84) Scemama, A.; Giner, E. An Efficient Implementation of Slater-Condon Rules. arXiv 2013,

(85) Sharma, S.; Holmes, A. A.; Jeanmairet, G.; Alavi, A.; Umrigar, C. J. Semistochastic Heat-Bath Configuration Interaction Method: Selected Configuration In- 
teraction with Semistochastic Perturbation Theory. $J$. Chem. Theory Comput. 2017, 13, 1595-1604.

(86) Peng, I. B.; Gokhale, M. B.; Green, E. W. System Evaluation of the Intel Pptane Byte-Sddressable NVM; ACM, 2019.

(87) DePrince, A. E.; Hammond, J. R. Coupled Cluster Theory on Graphics Processing Units I. The Coupled Cluster Doubles Method. J. Chem. Theory Comput. 2011, 7, 1287-1295.

(88) Kim, J.; Baczewski, A. D.; Beaudet, T. D.; Benali, A.; Bennett, M. C.; Berrill, M. A.; Blunt, N. S.; Borda, E. J. L.; Casula, M.; Ceperley, D. M. et al. QMCPACK: an Open Source ab initio Quantum Monte Carlo Package for the Electronic Structure of Atoms, Molecules and Solids. J. Phys.: Condens. Matter 2018, 30, 195901.

(89) Snyder, J. W.; Hohenstein, E. G.; Luehr, N.; Martínez, T. J. An Atomic Orbital-Based Formulation of Analytical Gradients and Nonadiabatic Coupling Vector Elements for the State-Averaged Complete Active Space Self-Consistent Field Method on Graphical Processing Units. J. Chem. Phys. 2015, 143, 154107.

(90) Ufimtsev, I. S.; Martínez, T. J. Graphical Processing Units for Quantum Chemistry. Comput. Sci. Eng. 2008, 10, 26-34.

(91) Kaliman, I. A.; Krylov, A. I. New Algorithm for Tensor Contractions on Multi-core CPUs, GPUs, and Accelerators Enables CCSD and EOM-CCSD Calculations with over 1000 Basis Functions on a Single Compute Node. J. Comput. Chem. 2017, 38, 842-853.

(92) Curtiss, L. A.; Raghavachari, K.; Referm, P. C.; Pople, J. A. Assessment of Gaussian-2 and Density Functional Theories for the Computation of Enthalpies of Formation. J. Chem. Phys. 1997, 106, 1063-1079.

(93) Schreiber, M.; Silva-Junior, M. R.; Sauer, S. P. A.; Thiel, W. Benchmarks for Electronically Excited States: CASPT2, CC2, CCSD and CC3. J. Chem. Phys. 2008, 128, 134110.

(94) Silva-Junior, M. R.; Schreiber, M.; Sauer, S. P. A.; Thiel, W. Benchmarks for Electronically Excited States: Time-Dependent Density Functional Theory and Density Functional Theory Based Multireference Configuration Interaction. J. Chem. Phys. 2008, 129, 104103.

(95) Sauer, S. P. A.; Schreiber, M.; Silva-Junior, M. R.; Thiel, W. Benchmarks for Electronically Excited States: A Comparison of Noniterative and Iterative Triples Corrections in Linear Response Coupled Cluster Methods: CCSDR(3) versus CC3. J. Chem. Theory Comput. 2009, 5, 555-564.

(96) Silva-Junior, M. R.; Sauer, S. P. A.; Schreiber, M.; Thiel, W. Basis Set Effects on Coupled Cluster Benchmarks of Electronically Excited States: CC3, CCSDR(3) and CC2. Mol. Phys. 2010, 108, 453-465.

(97) Silva-Junior, M. R.; Schreiber, M.; Sauer, S. P. A.; Thiel, W. Benchmarks of Electronically Excited States: Basis Set Effecs on CASPT2 Results. J. Chem. Phys. 2010, 133, 174318.

(98) Watson, T. J.; Lotrich, V. F.; Szalay, P. G.; Perera, A.; Bartlett, R. J. Benchmarking for Perturbative Triple-
Excitations in EE-EOM-CC Methods. J. Phys. Chem. A 2013, 117, 2569-2579.

(99) Kánnár, D.; Szalay, P. G. Benchmarking Coupled Cluster Methods on Valence Singlet Excited States. J. Chem. Theory Comput. 2014, 10, 3757-3765.

(100) Kánnár, D.; Tajti, A.; Szalay, P. G. Accuracy of Coupled Cluster Excitation Energies in Diffuse Basis Sets. J. Chem. Theory Comput. 2017, 13, 202-209.

(101) Watts, J. D.; Bartlett, R. J. Iterative and Non-Iterative Triple Excitation Corrections in Coupled-Cluster Methods for Excited Electronic States: the EOMCCSDT-3 and EOM-CCSD $(\tilde{T})$ Methods. Chem. Phys. Lett. 1996, 258, 581-588.

(102) Nooijen, M.; Bartlett, R. J. A New Method for Excited States: Similarity Transformed Equation-Of-Motion Coupled-Cluster Theory. J. Chem. Phys. 1997, 106, 6441-6448.

(103) Christiansen, P. A.; McCullough, E. A. Numerical Hartree-Fock Calculations for $\mathrm{N}_{2}$, FH, and CO: Comparison with Optimized LCAO Results. J. Chem. Phys. 1977, 67, 1877-1882.

(104) Shu, Y.; Truhlar, D. G. Doubly Excited Character or Static Correlation of the Reference State in the Controversial $2^{1} \mathrm{~A}_{\mathrm{g}}$ State of Trans -Butadiene? J. Am. Chem. Soc. 2017, 139, 13770-13778.

(105) Barca, G. M. J.; Gilbert, A. T. B.; Gill, P. M. W. Excitation Number: Characterizing Multiply Excited States. J. Chem. Theory. Comput. 2018, 14, 9-13.

(106) Barca, G. M. J.; Gilbert, A. T. B.; Gill, P. M. W. Simple Models for Difficult Electronic Excitations. J. Chem. Theory. Comput. 2018, 14, 1501-1509.

(107) Send, R.; Kühn, M.; Furche, F. Assessing Excited State Methods by Adiabatic Excitation Energies. J. Chem. Theory Comput. 2011, 7, 2376-2386.

(108) Page, C. S.; Olivucci, M. Ground and Excited State CASPT2 Geometry Optimizations of Small Organic Molecules. J. Comput. Chem. 2003, 24, 298-309.

(109) Guareschi, R.; Filippi, C. Ground- and Excited-State Geometry Optimization of Small Organic Molecules with Quantum Monte Carlo. J. Chem. Theory Comput. 2013, 9, 5513-5525.

(110) Bousquet, D.; Fukuda, R.; Maitarad, P.; Jacquemin, D.; Ciofini, I.; Adamo, C.; Ehara, M. Excited-State Geometries of Heteroaromatic Compounds: A Comparative TD-DFT and SAC-CI Study. J. Chem. Theory Comput. 2013, 9, 2368-2379.

(111) Tuna, D.; Lu, Y.; Koslowski, A.; Thiel, W. Semiempirical Quantum-Chemical Orthogonalization-Corrected Methods: Benchmarks of Electronically Excited States. J. Chem. Theory Comput. 2016, 12, 44004422.

(112) Budzák, Š.; Scalmani, G.; Jacquemin, D. Accurate Excited-State Geometries: a CASPT2 and CoupledCluster Reference Database for Small Molecules. $J$. Chem. Theory Comput. 2017, 13, 6237-6252.

(113) Tajti, A.; Stanton, J. F.; Matthews, D. A.; Szalay, P. G. Accuracy of Coupled Cluster Excited State Potential Energy Surfaces. J. Chem. Theory Comput. 2018, 14, 5859-5869. 
(114) Tajti, A.; Szalay, P. G. Accuracy of Spin-ComponentScaled CC2 Excitation Energies and Potential Energy

Surfaces. J. Chem. Theory Comput. 2019, 15, 55235531. 